\title{
Kommentar zum Design von Logistiknetzwerken
}

\author{
Anmerkungen zum Beitrag \\ Rieck, Julia: Ein Framework für die Gestaltung grosser, internationaler \\ Transportnetze: Berücksichtigung von Netzwerkdesign und Tourenbildung
}

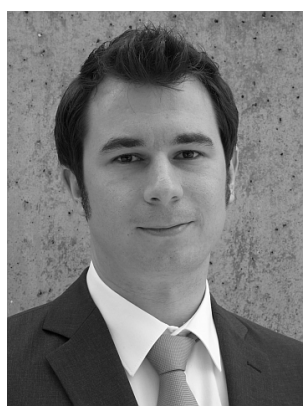

\section{Daniel Maucher}

\section{Einführung}

Das Design von Logistiknetzwerken ist bereits seit den siebziger Jahren Gegenstand wissenschaftlicher Untersuchungen (Geoffrion/ Powers 1995, 105). Auch die Festlegung der Anzahl und Standorte zentraler Umschlagspunkte wird schon seit den neunziger Jahren intensiv analysiert (Fleischmann/Gietz 2008, 141). Häufig findet dabei eine Kombination aus Standort- und Tourenplanung statt (Fischer 1997, 57). Im Zuge der fortschreitenden Globalisierung gewann ferner das Design von Logistiknetzwerken im globalen Kontext zunehmend an Bedeutung. Dennoch stehen sowohl Wissenschaft als auch Praxis bei der Planung von Transportnetzen immer noch vor grossen Herausforderungen. Dies gilt im besonderen Masse für die Konzeption internationaler Wertschöpfungsketten. Zunächst stellt sich beim Design von Logistiknetzwerken die Frage, welche Parameter bei der Bewertung berücksichtigt werden sollen. Zwar steigt mit der Anzahl der Parameter die Genauigkeit des Ergebnisses, doch gleichzeitig nimmt auch die Komplexität der Lösung zu, die dadurch häufig nicht in Polynomialzeit zu ermitteln ist. Eine weitere grosse Herausforderung ist dabei die praktische Erfassung der Datengrundlage und die Berücksichtigung ihrer möglichen Veränderungen. Als Beispiele hierfür dienen mögliche Veränderungen der Transportströme oder Veränderungen der Kostenstrukturen, wie z. B. Löhne, Treibstoffkosten und Wechselkurse.

\section{Inhalt des Beitrags}

Aufgrund der voranschreitenden Internationalisierung der Wertschöpfungsketten geht Rieck von einer wachsenden Bedeutung der Standort- und Transportplanung aus, zu deren Durchführung vor allem Beratungsunternehmen effiziente Werkzeuge benötigen. In ihrem Beitrag „Ein Framework für die Bewertung grosser, internationaler Transportnetze: Berücksichtigung von Netzwerkdesign und Tourenbildung“ stellt Rieck daher ein Modell zum Netzwerkdesign vor. Zunächst werden verschiedene Netzwerkkonfigurationen präsentiert und das Planungsproblem erläutert. Das Hub-Location-Problem wird anschliessend detailliert und in einem mathematischen Modell formuliert. Als Lösungsansatz für das Netzwerkdesignproblem wird ein heuristisches Verfahren vorgestellt, das sowohl für das Tourenplanungs- als auch für das Standortproblem eine Lösung erzeugt. Ein abschliessender Vergleich der Laufzeiten der exakten Lösung und des heuristischen Verfahrens zeigt, dass das exakte Verfahren bereits ab einer Anzahl von zwölf Knoten eine Lösung 
nicht mehr innerhalb einer halben Stunde erzeugen konnte. Mit dem heuristischen Verfahren dagegen konnte bei der Berechnung von praktischen Fallbeispielen mit teilweise über 100 Knoten i.d.R. in weniger als einer Stunde eine (sub-)optimale Lösung bestimmt werden.

\section{Relevanz des Beitrags}

Der Beitrag untersucht ein kombiniertes Standort- und Tourenplanungsproblem, bei dem Interdependenzen zwischen Standort- und Tourenplanung berücksichtigt und die Planung simultan durchgeführt wird. Konkret handelt es sich um eine integrierte Standort- und Tourenplanung. Dabei wird ein spezifisches Standortplanungsproblem mit einem spezifischen Tourenplanungsproblem kombiniert (Klose 2001, 293). Neben den exakten Verfahren werden zur Lösung der integrierten Standort- und Tourenplanung aufgrund der hohen Komplexität häufig Heuristiken verwendet, die in sequentielle, iterative und parallele Verfahren unterschieden werden. Während bei sequentiellen Verfahren die beiden Planungsschritte in beliebiger Reihenfolge nacheinander und ohne Rückkopplung erfolgen, nähern sich iterative Verfahren der optimalen Lösung durch wiederholtes Pendeln zwischen dem Standort- und Tourenplanungsproblem an. Bei der von Rieck vorgestellten Heuristik erfolgt die Lösung des Standortproblems im Rahmen der Tourenplanung, da Hubs ohne Touren gelöscht werden. Diese Heuristik ist daher den parallelen Verfahren zuzurechnen. Weitere parallele Verfahren wurden beispielsweise von Nambiar et al. (1981), Srivastaval Benton (1990), Srivastava (1993), Chien (1993), Nagy/Salhi (1996), Lin et al. (2002), Albareda-Sambola et al. (2005) sowie Melechovsky et al. (2005) formuliert (Nagy/Salhi 2007, $658 \mathrm{ff}$.). Da auch innerhalb der parallelen Verfahren zahlreiche Unterscheidungsmerkmale existieren, sind die Ausprägungen des Modells nach Rieck in Abbildung 1 dargestellt.

Die praktische Relevanz des Artikels zeigt sich besonders für Beratungsunternehmen, die häufig mit vielen unterschiedlichen Hub-Location-Problemen konfrontiert werden. Da die Heuristik auch bei hoher Knotenanzahl relativ schnell eine Lösung generiert, stellt diese und das Excel-basierte Entscheidungsunterstützungssystem besonders für zeitkritische Fragestellungen im Rahmen eines internationalen Supply Chain-Designs einen wertvollen Beitrag dar. 
Maucher | Kommentar zum Design von Logistiknetzwerken

\begin{tabular}{|c|c|c|c|c|c|c|c|c|}
\hline Merkmal & \multicolumn{8}{|c|}{ Ausprägung } \\
\hline Topographie & \multicolumn{2}{|c|}{ Ebene } & \multicolumn{3}{|c|}{ Netzwerk } & \multicolumn{3}{|c|}{ Diskrete Modelle } \\
\hline $\begin{array}{l}\text { Anzahl an Unterbre- } \\
\text { chungspunktebenen }\end{array}$ & \multicolumn{2}{|c|}{ Einstufig } & \multicolumn{3}{|c|}{ Zweistufig } & \multicolumn{3}{|c|}{ Mehrstufig } \\
\hline Art der Zielfunktion & \multicolumn{2}{|c|}{ Kostenminimierung } & \multicolumn{3}{|c|}{ Andere (z.B. Distanzen) } & \multicolumn{3}{|c|}{ Mehrere Zielfunktionen } \\
\hline $\begin{array}{l}\text { Ermittlung der Anzahl der } \\
\text { Hubs }\end{array}$ & \multicolumn{4}{|c|}{ Exogene Vorgabe } & \multicolumn{4}{|c|}{ Berechnung } \\
\hline Anzahl Produktgruppen & \multicolumn{4}{|c|}{ Einproduktmodell } & \multicolumn{4}{|c|}{ Mehrproduktmodell } \\
\hline $\begin{array}{l}\text { Kapazitierte vs. unkapazi- } \\
\text { tierte Einrichtungen }\end{array}$ & \multicolumn{4}{|c|}{ Kapazitierte Einrichtungen } & \multicolumn{4}{|c|}{ Unkapazitierte Einrichtungen } \\
\hline $\begin{array}{l}\text { Zuordnung der Nachfra- } \\
\text { gepunkte }\end{array}$ & \multicolumn{4}{|c|}{ Eindeutig } & \multicolumn{4}{|c|}{ Nicht eindeutig } \\
\hline Routenstruktur & \multicolumn{4}{|c|}{ Pendelbelieferung } & \multicolumn{4}{|c|}{ Belieferung durch Touren } \\
\hline Planungszeitraum & \multicolumn{4}{|c|}{ Eine Periode } & \multicolumn{4}{|c|}{ Mehrere Perioden } \\
\hline Art der Input-Daten & \multicolumn{3}{|c|}{ Deterministisch } & & \multicolumn{4}{|c|}{ Stochastisch } \\
\hline $\begin{array}{l}\text { Berücksichtigung besonde- } \\
\text { rer Tourenplanungsaspek- } \\
\text { te }\end{array}$ & $\begin{array}{l}\text { Mehrere } \\
\text { Fahrzeug- } \\
\text { typen }\end{array}$ & $\begin{array}{l}\text { Intermo- } \\
\text { dale } \\
\text { Trans- } \\
\text { portlösun- } \\
\text { gen }\end{array}$ & $\begin{array}{l}\text { Länder- } \\
\text { spezifi- } \\
\text { sche Tou- } \\
\text { ren }\end{array}$ & $\begin{array}{l}\text { Max. } \\
\text { Fahrleis- } \\
\text { tung bzw. } \\
\text {-dauer }\end{array}$ & $\begin{array}{l}\text { Zeit } \\
\text { ter a } \\
\mathrm{Nac} \\
\text { gep } \\
\mathrm{t}\end{array}$ & & $\begin{array}{l}\text { Zulassung } \\
\text { offener } \\
\text { Touren }\end{array}$ & $\begin{array}{l}\text { Länder- } \\
\text { spezifi- } \\
\text { sche Be- } \\
\text { sonderhei- } \\
\text { ten }\end{array}$ \\
\hline
\end{tabular}

Abbildung 1: Schematische Einordnung des Modells nach Rieck in die Verfahren zur parallelen Standort- und Tourenplanung

\section{Stärken und Schwächen der Methode}

Die Limitationen der vorgestellten Methode beziehen sich im Wesentlichen auf Beschränkungen hinsichtlich der Input-Daten und der getroffenen Annahmen des Modells. So wird durch die Verwendung einer repräsentativen Periode als Datenbasis von statischen Einflussparametern ausgegangen. Die Unsicherheit der zugrundeliegenden Daten wird daher nicht berücksichtigt und Standortentscheidungen können nicht im zeitlichen Ablauf erfolgen. Eine mehrmalige Durchführung der Heuristik mit Best-, Average- und Worst-Case Szenarien erscheint deshalb empfehlenswert. Ein Beispiel für Modellbeschränkungen stellt die Zugrundelegung eines einperiodischen Ansatzes dar. So werden bei der Berechnung zwar die zur Eröffnung der Hubs anfallenden Fixkosten sowie prognostizierte Unterhaltungskosten, nicht jedoch die Desinvestitionskosten der Hubs berücksichtigt. Weiterhin wird davon ausgegangen, dass Fahrzeuge im Planungszeitraum nur zweimal eingesetzt werden können. Die tatsächlich zurückzulegende Strecke der Fahrzeuge bleibt dabei unberücksichtigt. Auch die Nebenbedingungen, dass Quell- oder Zielknoten nur genau einmal angefahren werden dürfen, können ggf. einer operativen Lösung im Wege stehen. Sinnvoller wäre es, mehrfaches Anfahren zwar zu erlauben aber monetär zu bestrafen.

Die Stärken des Beitrags ergeben sich vor allem aus der simultanen Planung von Standorten und Transportwegen, wodurch ein Optimum aus Hub- und Transportkosten generiert werden kann. Da die Anzahl der Hubs nicht vorgegeben, sondern durch Berechnung erzeugt wird, kann diesbezüglich eine kostenoptimale Anzahl ermittelt werden. Ein weiterer Vorteil ergibt sich aus der Möglichkeit, Touren nur innerhalb eines Landes zuzulassen. Dadurch können Restriktionen des länderübergreifenden Verkehrs umgangen werden. 
Mögliche Erweiterungen des Modells können durch die Berücksichtigung der in Abbildung 1 genannten, besonderen Tourenplanungsaspekte beim Netzwerkdesign erfolgen. Beispiele hierfür sind die Einbeziehung intermodaler Transportlösungen sowie die Berücksichtigung länderspezifischer Besonderheiten, wie z. B. Zölle, Steuern und Gesetze.

\section{Literaturhinweise}

Albareda-Sambola, M., et al. (2005): A compact model and tight bounds for a combined locationrouting problem, in: Computers and Operations Research, Jg. 32, Nr. 3, S. 407-428.

Chien, T.W. (1993): Heuristic procedures for practical-sized uncapacitated location-capacitated routing problems, in: Decision Sciences, Jg. 24, Nr. 5, S. 995-1021.

Fischer, K. (1997): Standortplanung unter Berücksichtigung verschiedener Marktbedingungen, Heidelberg.

Fleischmann, B./Gietz, M. (2008): Transport- und Tourenplanung, in: Arnold, D., et al. (Hrsg.): Handbuch Logistik, 3. Aufl., Berlin/Heidelberg, S. 137-152.

Geoffrion, A.M./Powers, R.F. (1995): Twenty years of strategic distribution systems design: an evolutionary perspective, in: Interfaces, Jg. 25, Nr. 5, S. 105-127.

Klose, A. (2001): Standortplanung in distributiven Systemen, Heidelberg.

Lin, C.K.Y., et al. (2002): A location-routing-loading problem for bill delivery services, in: Computers and Industrial Engineering, Jg. 43, Nr. 1-2, S. 5-25.

Melechovský, J., et al. (2005): A metaheuristic to solve a location-routing problem with non-linear costs, in: Journal of Heuristics, Jg. 11, Nr. 5-6, S. 375-391.

Nagy, G./Salhi, S. (1996): Nested heuristic methods for the location-routing problem, in: Journal of the Operational Research Society, Jg. 47, Nr. 9, S. 1166-1174.

Nagy, G./Salhi, S. (2007): Location-routing: Issues, models and methods, in: European Journal of Operational Research, Jg. 177, Nr. 2, S. 649-672.

Nambiar, J.M., et al. (1981): A large scale location-allocation problem in the natural rubber industry, in: European Journal of Operational Research, Jg. 6, Nr. 2, S. 183-189.

Srivastava, R. (1993): Alternate solution procedures for the location-routing problem, in: Omega, Jg. 21, Nr. 4, S. 497-506.

Srivastava, R./Benton, W.C. (1990): The location-routing problem: Considerations in physical distribution system design, in: Computers in Operations Research, Jg. 17, Nr. 5, S. 427-435.

Daniel Maucher, Wirtsch.-Ing. M.Sc., ist wissenschaftlicher Mitarbeiter am Lehrstuhl für Logistikmanagement der Universität St.Gallen.

Anschrift: Universität St.Gallen, Lehrstuhl für Logistikmanagement, Dufourstrasse 40 a, CH-9000 St. Gallen, Tel.: +41 (0)71/224-7290, Fax: +41 (0)71/224-7315, E-Mail: daniel.maucher@unisg.ch 